\title{
Up-regulation of the vacuolar invertase TAI gene may contribute to the accumulation of carotenoids in tomato fruits
}

\author{
M.A. Slugina ${ }^{1 *}$, E.A. Dzhos ${ }^{1,2}$, A.V. Shchennikova ${ }^{1}$, E.Z. Kochieva ${ }^{1,3}$ \\ ${ }^{1}$ Institute of Bioengineering, Research Center of Biotechnology, RAS, Moscow, Russia \\ ${ }^{2}$ Lomonosov Moscow State University, Moscow, Russia \\ ${ }^{3}$ State Scientific Institution, All-Russian Research Institute for Selection and Seed Production of Vegetable Crops, RAS, Moscow, Russia
}

DOI 10.18699/ICG-PlantGen2019-47

(c) Autors, 2019

*e-mail: mashinmail@mail.ru

\begin{abstract}
Generally, fleshy fruit ripening is accompanied by its sweetening and color change caused by dramatic molecular, biochemical and physiological conversions. Species of the tomato clade differ in color of ripe fruits. Carotenoids accumulate in the fruit chromoplasts of red-fruited species, and the fruits of primitive green-fruited tomato species remain green due to the numerous blocks of carotenogenesis. In this study, the sugar and carotenoid content, as well as the expression patterns of the four genes encoding main fruit-specific sucrose cleavage enzymes TAl, SUS1, NI2 and LIN5, were characterized in immature green, mature green and ripe fruits of $S$. Iycopersicum cultivars and the wild green-fruited tomato species S. habrochaites and S. peruvianum. An analysis of the sugar and carotenoid content confirmed that carotenoids and hexoses accumulate in the ripe fruits of the red-fruited S. lycopersicum cultivars, while green-fruited tomatoes produce fruits enriched for sucrose and containing only trace amounts of carotenoids. The SUS1, LIN5 and NI2 expression showed no correlation with fruit color and hexose content. In fruits of green-fruited species, all analyzed genes were expressed in a similar way. TAl expression was extremely specific for cultivars, being maximal in ripe fruit, characterized by carotenoid and hexose accumulation in fruits. The findings suggest that vacuolar invertase TAI, rather than apoplastic invertase LIN5 or cytoplasmic sucrose synthase SUS1 and invertase $\mathrm{NI2}$, may specify sugar composition in ripe fruits.
\end{abstract}

Key words: sucrose hydrolysis genes; red- and green-fruited tomato species; carotenoid biosynthesis.

\section{Introduction}

Solanum section Lycopersicon includes the cultivated tomato S. lycopersicum and its 12 wild relatives that differ significantly in fruit physiology, biochemistry and morphology (Peralta et al., 2008). This makes tomato species an attractive model for studying the mechanism of fleshy fruit development from initiation to full maturity.

Ripening, converting mature fruit from inedible to edible form, is an irreversible process associated with perceptible organoleptic and visible alterations in texture, flavor, aroma, color, and size, due to dramatic molecular, metabolic, biochemical and physiological changes driven by internal and external signaling (Seymour et al., 2013). Ripening determines crop yield, shelf life, and fruit quality traits, including dry matter amount, content, and nutritional value (Li, Van Eck, 2007). The recently evolved so-called red-fruited tomato species (S. lycopersicum, S. pimpinellifolium, S. cheesmaniae, $S$. galapagense) have a yellow to red color of ripe fruits due to carotenoid synthesis (mainly beta-carotene and lycopene) and chlorophyll degradation (Llorente et al., 2016). Ripe fruits, formed by more ancient green-fruited tomato species, do not accumulate carotenoids and, as a result, stay green (Kilambi et al., 2017). The basic mechanisms of ripening are similar in both sets of tomato species and accompanied by high ethylene biosynthesis, fruit softening, and sugar content increment. However, they differ in sugar composition in ripening fruits: red-fruited species accumulate glucose and fructose, and green-fruited tomatoes contain sucrose (Beckles et al., 2012). The exact molecular mechanisms of mutual regulation of sugar metabolism and fruit pigmentation have not yet been fully understood. There is some evidence that sugars can act as signaling molecules to activate carotenoid biosynthesis and, as a result, the fruit acquires a different color. For example, changing the sugar concentration in mandarin (Citrus unshiu), watermelon (Citrullus lanatus) and tomato fruits alters their pigmentation (Iglesias et al., 2001; Telef et al., 2006; Zhang et al., 2017). Thus, a connection can be proposed between the sucrose-hexose ratio and the synthesis of carotenoids. In carbohydrate metabolism, glucose is the central molecule, sucrose is the most common form of translocated sugar, and sucrose-hexose interconversion is one of the main reactions (Winter, Huber, 2000). In plants, four sucrose cleavage enzymes are known: cytoplasmic sucrose synthase (SUS), and three invertases, acid vacuolar (TAI), acid apoplastic (LIN) and neutral cytoplasmic (NI) (Winter, Huber, 2000).

In this study, new data were obtained on the possible relationships between carbohydrate and carotenoid metabolism by analyzing fruit-specific expression patterns of SUS1, TAI, LIN5 and NI2 and fruit biochemical composition (sugars, carotenoids) during ripening in green- and red-fruited tomato species.

\section{Materials and methods}

Accessions of cultivated (S. lycopersicum cv. Silvestre Recordo, Red Cherry and Fioletovii) and wild (S. habrochaites and S. peruvianum) tomato species (Solanum section Lycopersicon) were provided by the N. I. Vavilov Institute of Plant Genetic Resources (St.-Petersburg, Russia) and grown in a greenhouse with a $16-\mathrm{h} / 8-\mathrm{h}\left(28{ }^{\circ} \mathrm{C} / 23^{\circ} \mathrm{C}\right)$ light-dark cycle (light intensity, 300-400 $\mu \mathrm{mol} \mathrm{m}^{-2} \mathrm{~s}^{-1}$ ). 
Total RNA was extracted from immature green (IF), mature green (MF) and fully ripe (RF) fruits using the RNeasy Plant Mini Kit (QIAGEN, Hilden, Germany) and analyzed by gel electrophoresis and fluorimetry (Qubit ${ }^{\circledR}$ Fluorometer, Thermo Fisher Scientific, Waltham, MA, USA). First-strand cDNA was synthesized using the Reverse Transcription System (Promega, Madison, WI, USA) and an oligo-dT primer and quantified by fluorimetry. Gene-specific primers separated by at least one big intron were designed to amplify fragments of coding sequences: SUSrtF (5'-GATTTCGAGCCTTTCACTGC-3')/SUSrtR (5'-AGGTATTCCTCTGCCTTCC-3') (for SUS1 gene), TAIrtF (5'-GAGGCTCCGGGAGTTGGTAA-3')/TAIrtR (5'-CC AAATCTTGACGGAGGCAG-3') (for TAI), LIN5rtF (5'-TGTCAAGAATGTTCATAGAACTC-3')/LIN5rtR (5'-TGAATGAGCCCAAATAATATTGC-3') (for LIN5) and NI2rtF (5'-ACTCTTGCTGCTAATGATCCTAA-3')/ NI2rtR (5'-ACTTCTTCATATTTGTTATCATCGAG-3') (for NI2). Quantitative real-time PCR (qPCR) was performed in two biological and three technical replicates using $2.5 \mathrm{ng}$ of cDNA, developed primers, and SYBR Green and ROX RT-PCR mixture (Syntol, Moscow, Russia) at the following conditions: denaturation at $95^{\circ} \mathrm{C}$ for $5 \mathrm{~min}$, and 40 cycles at $95^{\circ} \mathrm{C}$ for $15 \mathrm{~s}$ and $60^{\circ} \mathrm{C}$ for $40 \mathrm{~s}$. The expression levels were normalized to that of the tomato reference genes Expressed and Actin 2/7 (Expósito-Rodríguez et al., 2008; Bemer et al., 2012). Statistical analysis was performed using Graph Pad Prism version 7.02 (San Diego, CA, USA; https://www. graphpad.com/scientifc-software/prism/).

Biochemical analysis was performed in two biological replicates. To assess sucrose, glucose and fructose content, 1 $\mathrm{g}$ of fruit material was ground in liquid nitrogen, diluted with $10 \mathrm{ml}$ of $80 \%$ ethanol, centrifuged at 10,000 rpm for $15 \mathrm{~min}$, and analyzed by HPLC (Varian ProStar, VARIAN INC., Palo Alto, CA, USA) using an HPLC Refractive Index Detector 102M (Stayer, Moscow, Russia) and an Agilent Carbohydrate Analysis Column $(150 \mathrm{~mm} \times 4.6 \mathrm{~mm}, 5 \mu \mathrm{M})$; isocratic elution was performed with $75 \% \mathrm{v} / \mathrm{w}$ acetonitrile as a mobile phase; the flow rate was maintained at $1.5 \mathrm{ml} / \mathrm{min}$ and the temperature, at $30{ }^{\circ} \mathrm{C}$. Total carotenoids content was measured by Nagata and Yamashita equation (Nagata, Yamashita, 1992).

\section{Results and discussion}

Three $S$. lycopersicum cultivars and two wild tomato species, $S$. habrochaites and $S$. peruvianum, considered in this study represent two diverse groups varying in fruit pigmentation and sugar composition (Table 1). The same fruit samples of examined accessions were taken at three time points, including growth (IF), mature (MF) and ripe (RF) stages, both for biochemical and qPCR analyses.

An analysis of the sugar and carotenoid contents confirmed that carotenoids and hexoses accumulate in ripe fruits of the red-fruited $S$. lycopersicum cultivars, while green-fruited tomatoes produce fruits enriched for sucrose and containing trace amounts of carotenoids (see Table 1). The obtained data are fully consistent with the previously observed carbohydrate biochemistry of fruits in red- and green-fruited tomatoes (Peralta et al., 2008; Beckles et al., 2012) and suggest a negative correlation between the accumulation of monosaccharides and the presence of carotenoids.
To find out the possible factors influencing the difference in carbohydrate composition and, likely, in the implementation of the carotenoid biosynthesis in tomato fruits, SUS1, TAI, LIN5 and NI2 expression patterns were determined and compared in ripening fruits of red-fruited cultivars Silvestre Recordo, Red Cherry and Fioletovii and the green-fruited species $S$. habrochaites and S. peruvianum (Figure 1). These genes were selected for analysis among their paralogs because their products were shown to have fruit-specific activity (Winter, Huber, 2000; Baxter et al., 2005).

The transcription levels of the analyzed genes varied considerably relative to each other (see Figure 1). The SUS1, LIN5 and NI2 transcripts were found in fruits of both red- and green-fruited tomatoes, which indicates the absence of any correlation of their expression with fruit color.

In fruits of green-fruited species, all analyzed genes were expressed in a similar way. The LIN5 transcription level was the highest in mature green fruit and absent in ripe fruit. SUS1 transcription was maximal in immature green fruits and decreased during the transition from stage IF to stage RF.

Our observations suggest that primitive green-fruited species may have retained an ancestral expression pattern for each analyzed sucrose cleavage gene, as was also demonstrated for carotenoid metabolism genes (Kilambi et al., 2017).

In contrast, the SUS1 and LIN5 transcription varied between cultivars of the red-fruited species without correlation with the sugar and carotenoid content in ripe fruit.

Thus, S. lycopersicum cultivars, which, due to intensive tomato breeding, differ significantly in fruit color, sweetness and other related traits, may have diversified expression of sucrose catabolism genes with a unique pattern for each cultivar.

Unlike these genes, TAI expression was extremely specific to cultivars, being maximal in ripe fruit, characterized by carotenoid and hexose accumulation in fruits. Our observation is consistent with previous data on TAI expression in ripe fruits of other red-fruited tomato species (Slugina et al., 2017).

TAI activity in the vacuole may lead to accumulation of symplastic glucose in the cell, which may stimulate chromoplast formation, as shown for watermelon (Zhang et al., 2017).

Differences in fruit color in tomato species and cultivars may indicate the uniqueness of carotenoid biosynthesis regulation in each genotype.

Fruits of red-fruited species accumulate carotenoids at high levels in carotenoid sequestering substructures inside chromoplasts (Li, Van Eck, 2007). Fruit carotenogenesis coincides with the chloroplast into chromoplast transformation (Kilambi et al., 2017). In green-fruited species, fruits do not have orange/red color due to the numerous blocks of fruit-specific chromoplast formation and carotenoid synthesis (Kilambi et al., 2017).

\section{Conclusions}

The findings suggest that vacuolar invertase TAI, rather than apoplastic invertase LIN5 or cytoplasmic proteins, sucrose synthase SUS1 and invertase NI2, may be a key enzyme in determining sugar composition in ripe fruit. The role of cytoplasmic and apoplastic sucrose hydrolysis probably consists in maintaining the basic sucrose-hexose balance in the cells and in supplying dividing and expanding cells with energy 
Table 1

Sugar and carotenoid content in immature green (IF), mature green (MF) and ripe (RF) fruits of S. lycopersicum cultivars and two wild tomato species

\begin{tabular}{|c|c|c|c|c|c|}
\hline Accession & Fruit stage & $\begin{array}{l}\text { Glucose, } \mathrm{mg} / \mathrm{g} \text { fresh } \\
\text { weight }\end{array}$ & $\begin{array}{l}\text { Fructose, } \mathrm{mg} / \mathrm{g} \text { fresh } \\
\text { weight }\end{array}$ & $\begin{array}{l}\text { Sucrose, } \mathrm{mg} / \mathrm{g} \text { fresh } \\
\text { weight }\end{array}$ & $\begin{array}{l}\text { B-carotene, } \mathrm{mg} / \mathrm{g} \\
\text { fresh weight }\end{array}$ \\
\hline \multirow[t]{3}{*}{ S. habrochaites } & IF & 0.313 & 0.373 & 0.174 & \\
\hline & MF & 0.3865 & 0.493 & 0.4375 & \\
\hline & RF & 0.2285 & 0.339 & 1.5845 & $<0.001$ \\
\hline \multirow[t]{3}{*}{ S. peruvianum } & IF & 0.551 & 0.749 & 0.246 & \\
\hline & MF & 0.628 & 0.842 & 0.3125 & \\
\hline & RF & 0.024 & 0.399 & 1.328 & $<0.001$ \\
\hline \multirow{3}{*}{$\begin{array}{l}\text { S. lycopersicum cv. } \\
\text { Silvestre Recordo }\end{array}$} & IF & 0.837 & 0.812 & 0.181 & \\
\hline & MF & 0.9885 & 1.2075 & 0.149 & \\
\hline & RF & 1.3205 & 1.702 & 0.099 & 0.098 \\
\hline \multirow{3}{*}{$\begin{array}{l}\text { S. lycopersicum cv. } \\
\text { Red Cherry }\end{array}$} & IF & 0.916 & 0.952 & 0.172 & \\
\hline & MF & 0.818 & 0.917 & 0.201 & \\
\hline & RF & 0.893 & 1.203 & 0.055 & 0.08 \\
\hline \multirow{3}{*}{$\begin{array}{l}\text { S. lycopersicum cv. } \\
\text { Fioletovii }\end{array}$} & IF & 0.938 & 0.998 & 0.096 & \\
\hline & MF & 0.987 & 1.160 & 0.184 & \\
\hline & RF & 1.069 & 1.324 & 0.044 & 0.091 \\
\hline
\end{tabular}

a

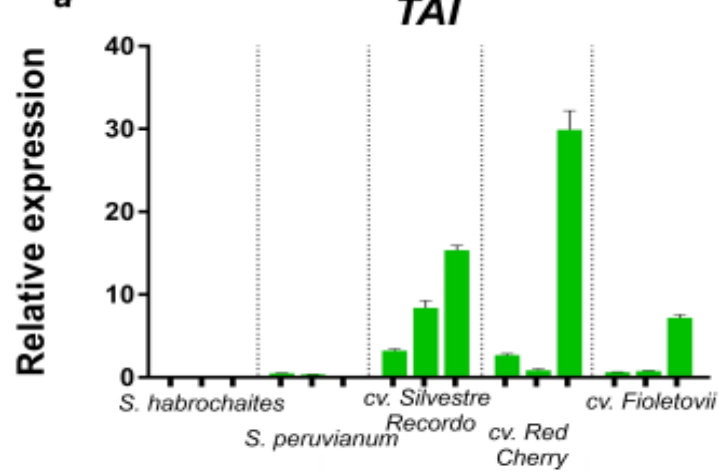

$c$

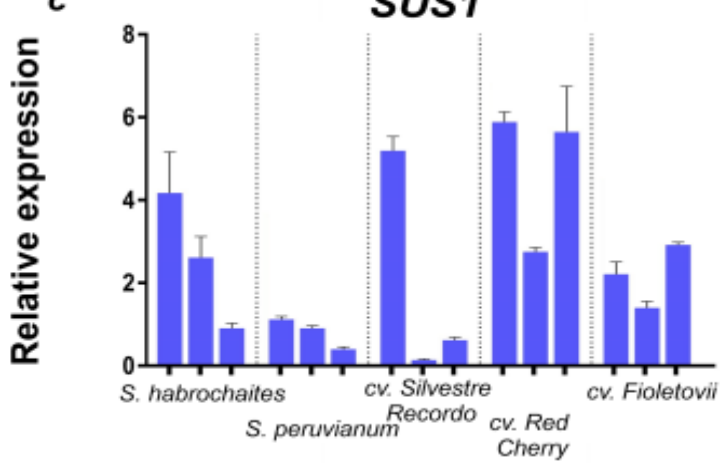

b

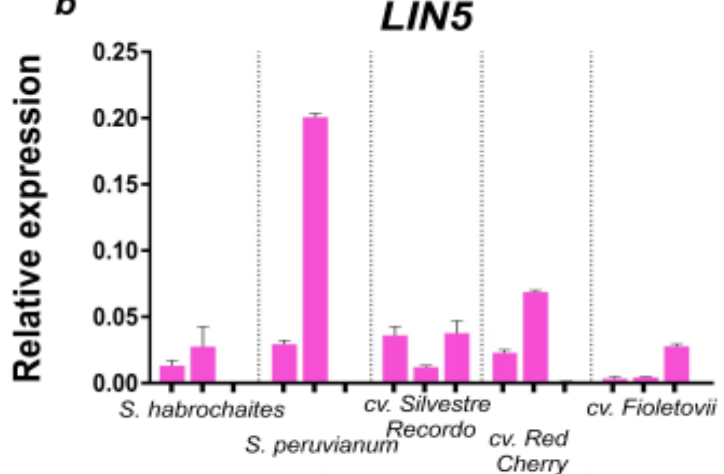

N/2

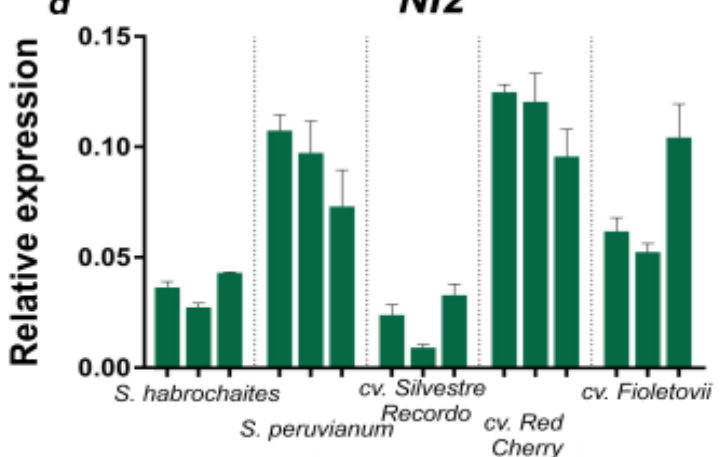

Figure 1. Expression patterns of TAI (a), LIN5 (b), SUS1 (c) and NI2 (d) in immature green, mature green and ripe fruits of S. lycopersicum cultivars and two wild tomato species. 
and carbon to synthesize the necessary compounds during fruit growth up to the stage of maturation.

Given the available evidence of a positive correlation between the sugar content and carotenoids in fruits, studying the genetic networks of fruit-specific carbohydrate metabolism may contribute to new alternative approaches to the manipulation of carotenoid levels in food crops.

\section{References}

Baxter C.J., Carrari F., Bauke A., Overy S., Hill S.A., Quick P.W., Fernie A.R., Sweetlove L.J. Fruit carbohydrate metabolism in an introgression line of tomato with increased fruit soluble solids. Plant Cell Physiol. 2005;46(3):425-437. DOI 10.1093/pcp/pci040.

Beckles D.M., Hong N., Stamova L., Luengwilai K. Biochemical factors contributing to tomato fruit sugar content: a review. Fruits. 2012;67:49-64. DOI 10.1051/fruits/2011066.

Bemer M., Karlova R., Ballester A.R., Tikunov Y.M., Bovy A.G., Wolters-Arts M., Rossetto P. de B., Angenent G.C., de Maagd R.A. The tomato FRUITFULL homologs TDR4/FUL1 and MBP7/FUL2 regulate ethylene-independent aspects of fruit ripening. Plant Cell. 2012;24(11):4437-4451. DOI 10.1105/tpc.112.103283.

Bolger A., Scossa F., Bolger M.E., Lanz C. et al. The genome of the stress-tolerant wild tomato species Solanum pennellii. Nature Gen. 2014;46:1034-1038. DOI 10.1038/ng.3046.

Expósito-Rodríguez M., Borges A., Borges-Pérez A., Pérez A. Selection of internal control genes for quantitative real-time RT-PCR studies during tomato development process. BMC Plant Biol. 2008;8:131. DOI 10.1186/1471-2229-8-131.

Iglesias D.J., Tadeo F.R., Legaz F., Primo-Millo E., Talon M. In vivo sucrose stimulation of colour change in citrus fruit epicarps: Interactions between nutritional and hormonal signals. Physiol. Plant. 2001;112(2):244-250. DOI 10.1034/j.1399-3054.2001.1120213.x.

Kilambi H.V., Manda K., Rai A., Charakana C., Bagri J., Sharma R., Sreelakshmi Y. Green-fruited Solanum habrochaites lacks fruit-specific carotenogenesis due to metabolic and structural blocks. $J$ Exp Bot. 2017;68(17):4803-4819. DOI 10.1093/jxb/erx288.

Li L., Van Eck J. Metabolic engineering of carotenoid accumulation by creating a metabolic sink. Transgen Res. 2007;16(5):581-585. DOI 10.1007/s11248-007-9111-1.
Llorente B., D'Andrea L., Ruiz-Sola M.A., Botterweg E., Pulido P., Andilla J., Loza-Alvarez P., Rodriguez-Concepcion M. Tomato fruit carotenoid biosynthesis is adjusted to actual ripening progression by a light-dependent mechanism. Plant J. 2016;85(1):107-119. DOI 10.1111/tpj.13094.

Nagata M., Yamashita I. Simple method for simultaneous determination of chlorophyll and carotenoids in tomato fruit. J. Japan. Soc. Food Sci. Technol. (Nippon Shokuhin Kogyo Gakkaishi). 1992; 39(10):925-928. DOI 10.3136/nskkk1962.39.925.

Peralta I.E., Spooner D.M., Knapp S. Taxonomy of Wild Tomatoes and their Relatives (Solanum sect. Lycopersicoides, sect. Juglandifolia, sect. Lycopersicon; Solanaceae. Systematic Bot Monographs. 2008; 84:1-186. ISBN 978-0-912861-84-5.

Seymour G.B., Østergaard L., Chapman N.H., Knapp S., Martin C. Fruit development and ripening. Ann Review Plant Biol. 2013;64: 219-241. DOI 10.1146/annurev-arplant-050312-120057.

Slugina M.A., Shchennikova A.V., Kochieva E.Z. TAI vacuolar invertase orthologs: the interspecific variability in tomato plants (Solanum section Lycopersicon). Mol. Gen. Genomics. 2017;292(5):11231138. DOI 10.1007/s00438-017-1336-y.

Télef N., Stammitti-Bert L., Mortain-Bertrand A., Maucourt M., Carde J.P., Rolin D., Gallusci P. Sucrose deficiency delays lycopene accumulation in tomato fruit pericarp discs. Plant Mol. Biol. 2006; 62:453-469. DOI 10.1007/s11103-006-9033-y.

Winter H., Huber S.C. Regulation of sucrose metabolism in higher plants: localization and regulation of activity of key enzymes. Crit. Rev. Biochem. Mol. Biol. 2000;35(4):253-289. DOI 10.1080/ 10409230008984165.

Zhang J., Guo S., Ren Y., Zhang H., Gong G., Zhou M., Wang G., Zong M., He H., Liu F., Xu Y. High-level expression of a novel chromoplast phosphate transporter CIPHT4;2 is required for flesh color development in watermelon. New Phytol. 2017;213(3):1208-1221. DOI 10.1111/nph.14257.

Acknowledgements. The work was supported by the Russian Science Foundation (grant No. 19-16-00016) and the Ministry of Science and Higher Education of the Russian Federation, and was performed using the experimental climate control facility (Institute of Bioengineering, Research Center of Biotechnology, Russian Academy of Sciences).

Conflict of interest. The authors declare no conflict of interest. 